\title{
ACERCA DE
}

Este artículo es una discusión de algunos de los usos ordinarios de "acerca de". Está dividido en dos secciones; la primera trata de un "dilema" que Nelson Goodman ha propuesto en un artículo del mismo título, ${ }^{1}$ en ella me interesa principalmente mostrar que el mentado "dilema" es espurio. La segunda sección consiste en un análisis preliminar de algunos aspectos de los usos ordinarios de 'acerca de', principalmente en relación con enunciados.

\section{I}

Enunciado brevemente, el "dilema" que Goodman propone es el siguiente: "Dado cualquier enunciado, podemos argüir, con plausibilidad, que es acerca de Maine. Por otro lado, admitir que todo enunciado es acerca de Maine es convertir en enteramente inútil cualquier afirmación acerca de que un enunciado dado es o no acerca de algo en particular". ${ }^{2}$ El "dilema" surge, señala Goodman, porque "aparentemente hablamos acerca de Maine siempre que hablamos de algo contenido en ... Maine, y siempre que hablamos acerca de cualquier cosa que contiene a Maine". 3 No es bastante claro lo que Goodman desea mostrar por medio de este "dilema"; puesto que la expresión "acerca de" en los ejemplos que él cita, y que son los que parecen dar origen al "dilema", es usada en sentido ordinario; podría pensarse por tanto que él quiere mostrar que ese sentido ordinario o uso de la expresión es responsable del "dilema". Esta explicación se hace plausible por el hecho de que antes afirma de las nociones ordinarias relacionadas con 'acerca de' que "fácilmente se puede mostrar que son inconsistentes". Sin embargo hay indicaciones en el artículo acerca de que, para él, el "dilema" surge de la confusión o identificación de dos diferentes usos o sentidos (¿ordinarios?) de 'acerca de'; porque de acuerdo con él, el escape al "dilema" reside en distinguir entre dos casos: "el uno, el de un enunciado que sea independiente o absolutamente acerca de una cosa dada; el otro, el de un enunciado que sea, en relación con otros ciertos enunciados, acerca de una cosa dada".4

Si esta última interpretación del intento de Goodman es correcta, nuestra primera tarea es descubrir si proviene de la forma en que 'acerca de' es usada ordinariamente. Tomemos los primeros cinco de los propios enunciados de Goodman: 1) "Maine tiene muchos lagos", 2) "Aroostook County está en Maine", 3) "En Aroostook County se cultivan patatas", 4) "Nueva Ingla-

1 Mind, vol. LXX, n9277, enero de 1961, pp. 1-24.

2 Ibid., p. 3.

3 Ibid., p. 2.

4 Ibid., p. 3 cursivas en el original. 
terra está al norte de Pennsylvania", y 5) "Los Estados de Nueva Inglaterra son pequeños". 1) es obviamente acerca de Maine y acerca de lagos, 2) es también acerca de Maine y acerca de Aroostoock County, mientras que de 3), 4) y 5) no se dice ordinariamente, sin especificaciones, que sean acerca de Maine, en el mismo sentido de 'acerca de' de 1) y 2). Esto es, estos tres enunciados no son, en ningún sentido ordinario, directamente acerca de Maine; pero pienso que nosotros por lo general diríamos que son indirecta o implicitamente (pero no afirman nada) acerca de Maine, mientras que 1) y 2) explícitamente afirman algo de él. Ahora, aunque 'implícitamente' e 'implica' son análogos, sus usos difieren en formas significativas en relación con la presente discusión. Si un enunciado $S$ es indirecta o implícitamente acerca de algún objeto, persona, etc,, $\mathrm{O}$, necesariamente implica algún hecho o presunto hecho acerca de $O$; por ejemplo, más arriba, 3) es indirectamente acerca de Maine e implica que en Maine se cultivan patatas, pero no creo que digamos que sea indirecta o implícitamente acerca del hecho de que en Maine se cultivan patatas. Por otro lado, con frecuencia muchos enunciados son directamente acerca de (puesto que afirman) ciertos hechos o presuntos hechos.

Al mismo tiempo, el concepto de implicación (en el presente uso ordinario de 'implica') es más amplio que el concepto de referencia indirecta de un enunciado, su indirecta "acercacidad"; toda clase de cosas pueden ser implicadas por un enunciado $S$ las cuales no diríamos ordinariamente que son (también) cosas acerca de las que $S$ es indirectamente acerca de. Por ejemplo, yo creo que, en el presente sentido, "Hoy es jueves" implica que mañana será viernes, pero no es indirectamente (y desde luego no directamente) açerca de ese hecho o presunto hecho. Asimismo, el enunciado "Jane Austin escribió Emma", implica que 'Jane Austin' es el nombre de la autora de Emma; "Jane Austin escribió Emma" implica " 'Jane Austin' es el nombre del ser (persona, animal, dios, etc.) que escribió Emma", pero ese enunciado no es indirecta, ni parcial ni totalmente, acerca del nombre 'Jane Austin' (o, en ese caso, el nombre 'Emma'). ${ }^{5}$ En forma similar "César fue asesinado" no es indirectamente acerca del nombre 'César', aunque lo que resulta ser el hecho -que alguien con el nombre de César fue asesinado- es válida-

-5 Cf. G. E. Moore a propos de "Mira, ése es Baldwin"; «me parece que en la inmensa mayoria de los casos en los que se usan nombres propios, tanto en la vida común, como en la historia y en ficción, nada se dice acerca del nombre usado; sólo está siendo usado. Esto es, la proposición expresada por la oración en la que aparece el nombre $\mathrm{N}$, no dice ni implica la proposición: "Alguien se llama ' $N$ '". Desde luego, al usar el nombre implicamos que alguien se llama " $N$ ", pero no en el sentido de que la proposición que expresamos implica esta proposición. Ryle evidentemente opina de otra manera. Piensa que si digo ahora "Baldwin es más bien un hombre bajo" esta oración implica (él hasta dice: "significa") lo que se significa con "Alguien llamado 'Baldwin' es más bien un hombre bajo". Pero no puedo evitar pensar que esto es un completo error.» (Philosophical Papers, Nueva York, 1962, pp. 107-108. Cursivas en el original.) 
mente inferible de él, es implicado por él. Finalmente, "La suegra es la madre del cónyuge" no res, indirectamente, acerca de las palabras 'suegra' y 'madre del cónyuge', pero implica que, en español 'suegra' y 'madre del cónyuge' son sinónimos, que 'suegra' quiere decir "madre del cónyuge".

Si lo que hemos dicho acerca de 3), 4) y 5) es verdad, se sigue que la primera premisa del argumento del que surge el dilema, es decir, que nosotros hablamos acerca de Maine (sin especificaciones) siempre que hablamos acerca de cualquier cosa contenida en él (llámese $M_{1}$ ), es falsa, ya que descansa en la falsa asunción de que 3) es, sin especificaciones, acerca de Maine, y la proposición de que 3) es, sin especificaciones, acerca de Maine es ella misma inferida del enunciado verdadero 2), junto con la asunción implicada de que un enunciado acerca de algo es (también), sin especificaciones o en el mismo sentido, acerca de aquello que lo contiene (llámese "principio" $\mathrm{P}_{1}$ ). Puesto que la conclusión falsa " "En Aroostook County se cultivan patatas' es, sin especificaciones, acerca de Maine" es inferible válidamente de las anteriores premisas, al menos una de estas premisas debe ser falsa; pero puesto que la primera premisa, esto es, "Aroostook County está en Maine" es verdadera, se sigue que la otra premisa, esto es $\mathrm{P}_{1}$, es falsa.

Puesto que $P_{1}$ es la primera parte del "principio" (llámesele P) cuya adopción conduce al "dilema" de Goodman, debemos hablar aún más acerca de la distinción entre la referencia directa e indirecta de un enunciado, donde, para empezar, el enunciado es directamente acerca de una u otra cosa.

En su uso actual, ' $S$ es indirectamente acerca de $\mathrm{X}$ ', puede ser vertido como " $S$ hace una referencia indirecta a $\mathrm{X}$ ", o "refiere indirectamente a $\mathrm{X}$ ". Cuando alguien dice "Pickwick Papers es una obra humorística", el enunciado tiene una referencia indirecta, digamos el autor de Pickwick Papers; también, creo, implica que el autor de Pickwick Papers, quien quiera que haya sido, escribió una obra humorística. Vale la pena notar una diferencia interesante entre 'hablar (directa o indirectamente) acerca de' y 'referir (directa o indirectamente) a'. "A habla acerca de Y" presupone lógicamente "A se refiere a $\mathrm{Y}$ ", pero la conversa no es verdadera. Obviamente el referirse de A a $\mathrm{Y}$ es una condición, pero sólo una condición, del que $\mathrm{A}$ hable acerca de $Y$; por ejemplo, A (en algún enunciado $S$ ) puede referirse indirectamente a $\mathrm{Y}$, en cuyo caso no estará hablando acerca de $\mathrm{Y}$. Desde luego, uno también puede referirse a $\mathrm{Y}$ y (o, con objeto de) hacer toda clase de cosas diferentes con $\mathrm{Y}$. 'Hablar acerca de' puede ser propuesto ya sea en un sentido amplio o en un sentido restringido; en un sentido amplio cubre varias cosas: hablo acerca de $\mathrm{Y}$ si hago una pregunta acerca de $\mathrm{Y}$, doy una orden, hago una petición o una súplica con respecto a $\mathrm{Y}$, deseo algo para o con respecto a $Y$, y así sucesivamente. En el sentido restringido, yo sólo hablo acerca de $\mathrm{Y}$ si enuncio o afirmo algo acerca de $\mathrm{Y} .^{6}$

6 Pero nótese que cuando alguien, A, dice: "Pickwick Papers es una obra humorís- 
Me parece que un análisis de diversas clases de enunciados muestra que a) un enunciado que es directamente acerca de alge, $\mathrm{X}$ puede ser, indirectamente, acerca de aquello en que $X$ está contenido, acaece o se encuentra, constituye un elemento o parte de, y así sucesivamente si tal es el caso. En forma similar, $b$ ) un enunciado que es directamente acerca de algo $\mathrm{X}$ puede ser (aunque pienso que en la mayoría de los tipos de casos no lo es) también indirectamente acerca de cualquier cosa particular que esté contenida en X, acaezca en ella, o forme parte de ella, etc. Para sustanciar e ilustrar estos puntos consideraré algunos enunciados relevantes.

1) Considérese, primero, el enunciado "Esta caja (la que resulta que contiene canicas) es roja"; éste no es indirectamente acerca de las canicas que contiene la caja. En forma similar "Estas canicas están hechas de vidrio", refiriéndose a las canicas contenidas en la caja, no es un enunciado indirectamente acerca de la caja misma; la caja por un lado y las canicas por el otro son cosas distintas, objetos independientes que ocurre que se encuentran yuxtapuestos de una cierta forma en un cierto momento.

2) Por otro lado, "En Aroostook County se cultivan patatas" es, como se afirmó antes, indirectamente acerca de Maine, pero "En Maine se cultivan patatas" no es indirectamente acerca de Aroostook County o cualquier otro lugar específico en Maine en el que se cultiven patatas. Considérese de nuevo ahora "En Aroostook County se cultivan patatas"; este enunciado también es indirectamente acerca de Nueva Inglaterra, los Estados Unidos, el hemisferio norte, y aun del mundo entero, el que contiene a Aroostook County en el mismo sentido en el que Maine contiene a Aroostook County; pero no podemos decir con propiedad que dicho enunciado es indirectamente acerca del Sistema Solar o acerca de la Vía Láctea como un todo, ya que tanto el Sistema Solar como la Vía Láctea contienen a la Tierra, y por tanto a Aroostook County, en un sentido diferente de 'contener' del sentido en el que la Tierra, el hemisferio norte, el Nuevo Mundo, etc., contienen a Aroostook County; los primeros contienen a la Tierra puramente como una parte numérica, como uno entre los muchos cuerpos celestes que contienen, mientras que los últimos contienen a Aroostook County como una parte cuantitativa: Aroostook County es una porción de la.superficie de la Nueva Inglaterra, de la de los Estados Unidos y de la del globo terráqueo.

Por otro lado, del enunciado "La Tierra gira alrededor del Sol" o aun "La Tierra gira alrededor de sí misma" puede decirse con propiedad que es

tica", A puede tener al señor Pickwick, digamos, en mente, puede estar pensando en (o acerca de) él, aun cuando pueda no desear o tratar, indirectamente, de referirse a él en (o al hacer) su enunciado (asimismo, desde luego, el enunciado anterior no refiere indirecta, especificamente al señor Pickwick o algún otro personaje, incidente, etc., específico, de la obra, aunque algunos de los personajes y/o incidentes, etc., de Pickwick Papers deben, obviamente, ser humorísticos si el enunciado ha de ser verdadero). Asi, hay un sentido de 'tener en mente' o 'pensar en (o acerca de)' que es diferente a 'referirse a (indirectamente)' en el sentido (1). 
indirectamente acerca del Sistema Solar y por lo tanto también acerca de la Vía Láctea y aun del universo como un todo.

3) Considérese ahora: "Él es un hombre grande", este enunciado no es, indirectamente, acerca de ninguna parte particular del cuerpo del hombre; en forma similar "El tiene manos grandes" no es indirectamente acerca del cuerpo del hombre como un todo. Sin embargo, "Él está enfermo del hígado" es, indirectamente, acerca del cuerpo del hombre (como un todo); en forma similar "Él sólo tiene un riñón" parece ser indirectamente acerca del cuerpo del hombre (aunque creo que no diríamos que es acerca de su cuerpo "como un todo"). Así, si después de hacer alguno de estos enunciados, alguien me pregunta: ¿Acerca de qué estás hablando?, puedo, si no quiero especificar, replicar verazmente: "Acerca del cuerpo de Fulano de Tal." Pero, mientras que el primer enunciado es indirectamente acerca del cuerpo enfermo del individuo, no hay nada que corresponda estrechamente a "cuerpo enfermo" en el último caso que pueda ser considerado como el referente indirecto del enunciado.

4) Consideremos ahora "El sistema euclidiano es un sistema de geometría tridimensional", este enunciado no es, indirectamente, acerca de algún axioma o teorema en particular, sin embargo, es indirectamente acerca de todos y cada uno de los axiomas y teoremas en el sistema, puesto que el carácter tridimensional de la geometría euclidiana es una penetrante característica del sistema. Pero considérese ahora: "El axioma de juego limpio afirma que..." Esto claramente no es indirectamente acerca de ningún sistema particular, incluyendo a la geometría euclidiana; por una razón: el axioma de juego limpio puede aparecer en muchos sistemas diferentes de geometría tridimensional, no sólo de geometría euclidiana. No obstante que es una parte necesaria de la geometría euclidiana, tal y como la tenemos, no necesariamente existe sólo como una parte de ella.

5) Considérese ahora: "Él es un buen jugador de futbol" y "El equipo de Varsity es un buen equipo". El primer enunciado no es, indirectamente, acerca de ningún equipo del cual el jugador individual sea, o haya sido, miembro; sin embargo, lógicamente hablando, una persona sólo puede ser un jugador de futbol como miembro de algún equipo de futbol. Desde luego, podemos inferir de este enunciado que cualquier equipo de futbol en el que él juegue, será un equipo con un buen jugador, pero esto no constituye información alguna acerca del equipo como un todo. El único hecho que posiblemente podría ser inferido de aquí es que el equipo del. cual él es miembro es un buen equipo, pero tal inferencia cometería la falacia de la composición y no puede ser hecha válidamente.

Se ve fácilmente que si ninguna inferencia válida de ninguna clase puede ser hecha acerca de algo $\mathrm{Y}$ a partir de un enunciado particular $S$, no es posible que $S$ sea directa o indirectamente, parcial o totalmente, acerca de 
$Y$; pero partiendo de esto no podemos argüir de la siguiente manera: si podemos inferir válidamente algo acerca de $\mathrm{Y}$ a partir de $S$, entonces $S$ es directa o indirectamente acerca de $\mathrm{Y}$. Y, como hemos visto, este enunciado condicional mismo es falso. Se ve también en el caso de "El equipo de Varsity es un buen equipo". Este enunciado no es indirectamente acerca de ningún jugador o jugadores individuales en el equipo, pero no puede ser verdadero a menos que algunos - probablemente la mayoría - de los jugadores en él sean muy buenos o buenos jugadores. Esto quiere decir que a partir de "El equipo Varsity es bueno" podemos inferir válidamente (como una condición de la verdad del enunciado) que algunos (la mayoría) de sus miembros son buenos jugadores, y, sin embargo, el enunciado no es ni siquiera indirectamente acerca de algún jugador particular o algún subgrupo particular de jugadores en el equipo.

Hasta ahora hemos intentado mostrar, en efecto, que el "principio" de que cualquier enunciado es un enunciado acerca de Maine es falso - lo que nadie negaría - apelando a nuestro conocimiento del uso ordinario de 'acerca de' y señalando que ordinariamente no diríamos que los enunciados 3), 4) y 5) son (directamente) acerca de Maine. Podemos agregar ahora que el hablar acerca de uno de estos enunciados como siendo (directamente, sin especificaciones) acerca de Maine sería confundir o identificar los dos sentidos de 'acerca de' que hemos señalado, o extender el primero de estos sentidos aplicándolo a 3), 4) y 5); en cualquier caso, ello significaría alejarse del uso ordinario. Precisamente, lo absurdo del "principio" $\mathbf{P}$ es un síntoma dramático y resultado de este alejamiento; es un alejamiento del uso ordinario que vacía a 'acerca de' y su negación de todo significado posible al excluir todas las posibles aplicaciones, así como las reales, del último. $O$, como lo enuncia Goodman, el "principio" $P$ hace "completamente inútil cualquier afirmación acerca de que un enunciado dado sea o no acerca de algo en particular". ${ }^{7}$

Es interesante notar aquí que la reducción al absurdo que el dilema de Goodman encierra en efecto, es, en este caso particular, una consecuencia lógica de un alejamiento del uso ordinario de una clase diferente a la que los filósofos se refieren comúnmente como una violación de tipo o de categoría; porque, asumiendo que se puede hablar con propiedad de 'acerca de' como perteneciendo a algún tipo lógico o categoría sin ninguna extensión de esa noción, no parece que haya ninguna violación de tipo al hablar de 3), 4) o 5) como siendo acerca de Maine. Tenemos aquí un ejemplo de lo que en otra parte he llamado una violación "inter-tipo" ${ }^{8}$ de significado: una violación "inter-tipo" que, como todas las violaciones de (intra-) tipo,

7 Ibid.

8 En $A$ Critical Study in Method, La Haya, 1967. 
es puesta de manifiesto por un argumento de reducción al absurdo basado en el enunciado, que contiene la violación, como una de sus premisas.

\section{II}

Pasamos ahora a considerar algunos aspectos de ciertos usos ordinarios de 'acerca de'. Los tópicos a tratar son los siguientes: I) La relación lógica entre la sinonimia o no-sinonimia de dos o más enunciados y acerca de qué son o podrían ser. Esto nos conducirá a 2), el uso de 'acerca de' en enunciados de segundo o más alto orden. Algunos de los resultados a los que se llegue aquí serán aplicados entonces a enunciados que contengan las palabras 'verdadero' y 'falso'. Después pasaremos a 3), la pregunta acerca de si un enunciado y lo que él implica son o no, o pueden ser, acerca de las mismas cosas. Después pasaremos a 4), algunas observaciones relacionadas con la definición y el análisis, surgidas de nuestras consideraciones de la gramática lógica de 'acerca de'. Concluiremos nuestra discusión con 5) analizando brevemente el uso de "acerca de" en relación con oraciones, en contraste con su uso en relación con enunciados.

r) Es un hecho elemental el que si dos enunciados tienen el mismo significado, necesariamente serán acerca de las mismas cosas; por otro lado, dos enunciados pueden ser acerca de las mismas cosas y diferir en significado; si esto último no fuera verdad, habría sido imposible el tener más de una proposición verdadera acerca de cualquier cosa. Se sigue de aquí que el ser acerca de las mismas cosas - cuando de dos enunciados se puede decir con propiedad que son, o pueden ser, acerca de algo, real o imaginario- es una condición necesaria pero no suficiente para que dos enunciados tengan el mismo significado; lo mismo es verdad para todos los otros tipos de expresiones verbales que son, o pueden ser, acerca de una cosa u otra. Esto excluye a las palabras aisladas, incluyendo nombres comunes, y a todas las frases. Por lo general, no hablamos de la palabra 'mesa' como siendo, digamos, "acerca de" mesas, en vez de eso decimos que nombra, o que es el nombre de ciertos objetos que tienen tales y cuales caractrísticas y usos generales. En forma similar, nosotros no hablamos ordinariamente de, digamos, 'la torre Eiffel' o 'un rey de Francia' como siendo acerca de algo, sin excluir la torre Eiffel ni ningún rey de Francia, respectivamente. De aquí que las llamadas "descripciones definidas" y "descripciones indefinidas" no sean, en un vacuum contextual, realmente descripciones en ningún sentido ordinario, ${ }^{9}$ ya que ninguna expresión verbal puede ser una descripción de absolutamente

9 Sin embargo, cuando ocurren "predicativamente" en enunciados de la forma " $\mathrm{X}$ es un rey de Francia" o "Y es la torre Eiffel", tienen una función descriptiva. Formarán también parte de una descripción de $\mathrm{X} \circ \mathrm{Y}$ en enunciados tales como "X es un rey de Francia y es calvo" y "Y es la torre Eiffel y está hecha de acero, tiene tal o cual altura", $\mathrm{y}$ asi sucesivamente. 
nada sin ser, primero, acerca de una cosa u otra. Yo agregaría que sólo de las oraciones (y los enunciados), entre las expresiones verbales, se dice ordinariamente que son acerca de algo; sin embargo, no es ni siquiera verdad que de todos los tipos de oraciones se diga que son acerca de algo. Sólo las oraciones declarativas y las preguntas caen dentro de esta categoría; de órdenes, peticiones, ruegos, no se dice, ordinariamente, que sean acerca de algo, aunque claramente refieren a algo; por ejemplo, la acción particular que le es encomendada, requerida o pedida de llevar a cabo al que escucha.

El hecho de que dos enunciados no puedan ser sinónimos a menos de que sean acerca de las mismas cosas puede proveernos, teóricamente hablando, de un criterio de no-sinonimia, en relación con los tipos de expresiones verbales mencionadas un poco antes. Sin embargo, en la práctica real, su utilidad no puede ser muy grande, es muy posible que en la mayoría de los casos donde puede ser de hecho usado, no se necesite para nada. Puede ser que, en estos casos, podamos ver que dos enunciados dados son no-sinónimos sin antes observar si son o no acerca de las mismas cosas. Por otro lado, en caso de enunciados muy complejos, donde no es nada claro si son o no sinónimos, puede, igualmente, ser muy difícil el determinar si son o no acerca de las mismas cosas. Creo, sin embargo, que nuestro criterio no es completamente inútil, y ahora ilustraré su uso considerando algunos tipos de enunciados. En este transcurso descubriremos también otros puntos de interés en relación con la gramática lógica de 'acerca de'.

El primer tipo de enunciados a considerar son enunciados acerca de otros enunciados. Se puede mostrar fácilmente que tales enunciados no son, y no pueden ser, directamente acerca de lo que los segundos son o pueden ser directamente. Así, "César fue asesinado' es acerca de un gran romano” . $(S)$ es un enunciado acerca del enunciado "César fue asesinado" (y también acerca de un [algún] gran romano) $(C)$; mientras que el enunciado mismo "César fue asesinado" es directamente acerca de otra cosa, el que César haya sido asesinado.

A la luz de la distinción entre aquello acerca de lo cual es un enunciadó directamente y aquello de lo que es indirectamente acerca de, en el caso de enunciados que son o pueden ser acerca de una cosa u otra para empezar, nuestro criterio de no-sinonimia es ahora expresable en forma más precisa como sigue: dos expresiones verbales, de la clase relevante, son no-sinónimas si una no es directamente acerca de aquello de lo cual la otra es directamente acerca de y/o no es indirectamente acerca de aquello que la otra es indirectamente acerca de. Notemos que la última parte del criterio no puede ser eliminada sin pérdida; es verdad que las cosas de las cuales un enunciado es directamente acerca de, determinan lógicamente las clases de cosas de las que puede y las clases de cosas de las que no puede ser acerca de indirectamente. El hecho de que el enunciado "En Aroostook County se culti- 
van patatas" es directamente acerca de Aroostook County hace lógicamente posible el que sea indirectamente acerca de Maine, y el hecho de que sea directamente sólo acerca de Aroostook County y el que se cultiven patatas en Aroostook County (lo que se entiende normalmente que es el caso cuando decimos "En Aroostook County se cultivan patatas") hace que sea imposible para el enunciado el ser indirectamente acerca de, digamos, el Sistema Solar. Pero qué cosas particulares son instancias de las clases de cosas acerca de las cuales el enunciado puede o no puede ser acerca de indirectamente, es una cuestión de hecho, y no es determinada meramente por aquello acerca de lo cual el enunciado es directamente acerca de.

Puesto que $S$ y $C$ son directamente acerca de diferentes cosas, se sigue de nuestro criterio que son diferentes en significado; sin embargo, en este caso particular, y en casos similares, no se necesita ningún criterio semejante para saber esto, podemos ver inmediatamente que son diferentes en significado tan pronto como atendemos a $S$ y a $C$.

2) En relación con enunciados que emplean las palabras verdadero y falso, esta situación es bastante diferente; por ejemplo, el enunciado "El enunciado 'César fue asesinado' es verdadero" $(N)$; no es fácil determinar si $N$ significa o no lo mismo que el enunciado "César fue asesinado". De hecho, como es bien sabido, F. P. Ramsey, ha sostenido que $N$ "no significa otra cosa que" $C ;{ }^{10}$ de acuerdo con él, las frases en las que aparecen 'verdadero' y 'falso' "son frases que a veces usamos por razones estilísticas o de énfasis, o para indicar la posición ocupada por el enunciado en nuestro argumento". 11 En forma similar P. F. Strawson, siguiendo a Ramsey, ha argüido que verdadero y falso son palabras performativas más que descriptivas, y de aquí que las oraciones en las que aparecen, más que enunciar hechos, expresen actividades. De acuerdo con las circunstancias, nuestra finalidad al usar estas expresiones es autorizar, subrayar, confirmar o conceder lo que ha sido o podría haber sido dicho por otro.

Ahora bien, yo estoy de acuerdo con Ramsey y Strawson en la medida en que pienso que 'verdadero' y 'falso' tal y como son usadas ordinariamente en expresiones como 'es verdad...' y 'es verdad que...' y quizás también en otras expresiones, ciertamente tienen una función performativa. ${ }^{12}$ Pero el punto de vista mismo acerca de que tienen esta función performativa implica lógicamente que $N$ dice algo diferente, tiene un significado distinto de $C$; la razón es bastante simple, no podemos, por medio de un enunciado, autorizar, subrayar, confirmar o conceder el hecho de que César fue asesinado a

10 The Foundations of Mathematics, Londres, 1931, p. 142.

11 Ibid.

12 Me inclino también fuertemente a pensar que ésta es sólo una parte de su función ordinaria. Véase en relación con esto, W. H. Walsh, "A Note on Truth", Mind, vol. LXI, no. 241, enero de 1952 , pp. 72-74. Véase también mi "Truth", por aparecer en Man and World. 
menos de que el enunciado en el que hagamos tal cosa, afirme algo diferente al enunciado que (meramente) enuncia o afirma ese hecho; el último afirma o enuncia el hecho, mientras que el primero autoriza, subraya o confirma la afirmación del hecho, o implica que lo que es afirmado (y por tanto se piensa que es un hecho) es realmente un hecho. En efecto, digo que $N$ y $C$ son (directamente) acerca de cosas diferentes, de aquí el que sean diferentes en significado; porque $N$ es directamente acerca de $C$, mientras que $C$ es (directamente) acerca de que César haya sido asesinado. La diferencia aquí es la misma que entre "César fue asesinado", afirmado por alguien, y "Yo estoy de acuerdo en que César fue asesinado" afirmado por alguien que escucha o lee el primer enunciado, porque "Yo estoy de acuerdo en que César fue asesinado" es una forma diferente de enunciar lo que $N$ enuncia.

Supongamos que estamos de acuerdo con Strawson en que 'verdadero' y 'falso' en $N$ expresan actividades y no enuncian o ayudan a enunciar algo acerca del enunciado $C$; aún quedaría el que $N$ y $C$ son diferentes en significado. La palabra 'verdadero' (y lo mismo se aplica a la palabra 'falso') no puede expresar lo que expresa sin hacer que $N$, como un todo, sea diferente en significado de $C$, porque seguramente $C$ no expresa acuerdo, etc., con nada. Sin embargo no estoy identificando "expresar" con "significar".

La situación es algo diferente con "Yo sé que César fue asesinado" $(K)$, vis-á-vis $C$, porque el primero, $K$, debe ser parafraseado por "Yo sé que el enunciado 'César fue asesinado' es verdadero" $(M)$ y no puede ser parafraseado con propiedad por "El enunciado 'César fue asesinado' es verdadero" $(N)$, ni tampoco $K$ o $M$ implican lógicamente a $N$ o viceversa. Pero $N$ se sigue lógicamente de $K$ o $M$ si agregamos la premisa " $K$, o $M$, es verdadero"; esto es, $N$ será implicado por $K$ o $M$ siempre que el último sea verdadero. Por otro lado, $N$ en general no implica $K$ o $M$ aun donde él mismo sea verdadero; esto es, en general, no podemos inferir válidamente $K$ o $M$ de $N y$ " $N$ es verdadero"; sólo lo podemos hacer cuando el que profiere ambos enunciados es una y la misma persona.

3) Pasamos ahora a la pregunta acerca de si un enunciado y lo que él implica es directamente acerca de la misma cosa, asumiendo, para empezar, que el primero se refiere a algo. La respuesta parece ser afirmativa, aún más, un enunciado y lo que él implica son, en forma necesaria, directamente acerca de las mismas cosas. Esto puede ser visto fácilmente examinando varias formas de enunciados y tantos como podamos de los enunciados que ellos implican; esto también es de suponerse de nuestro conocimiento de la naturaleza de la implicación como una relación lógica.

Sin embargo, no se sigue de aquí el que un enunciado (directamente) acerca de otro enunciado sea también (directamente) acerca de aquello que ese enunciado implica. De hecho, de nuestra primera discusión de los usos de 'acerca de' en relación con enunciados acerca de otros enunciados, sabemos 
que es falso el que un enunciado (directamente) acerca de otro enunciado sea acerca de lo que el último implica. Un ejemplo es el enunciado " los hombres son mortales' expresa una proposición universal”, este enunciado es (directamente) acerca de "todos los hombres son mortales", pero no lo es también (directamente) acerca de "algunas cosas mortales son hombres" que el primero implica. Lo mismo se aplica para enunciados acerca de otros tipos de enunciados.

4) Pasamos ahora a la pregunta acerca de si un enunciado directamente acerca de alguna expresión verbal es (también) directa o indirectamente acerca de cualesquiera expresiones verbales que sean sinónimas de ella. Al tratar de responder a esta pregunta, un hecho nuevo e interesante surge en relación con los usos ordinarios de 'acerca de' en la presente relación. Se puede ver fácilmente que un enunciado directamente acerca de alguna expresión verbal no es (también) directamente acerca de ninguna expresión verbal que sea sinónima de ella; pero puede ser, dependiendo de su carácter específico, indirectamente acerca de alguna o algunas de tales expresiones. Algunos enunciados acerca de una expresión verbal son definitivamente considerados, en el discurso ordinario, como indirectamente acerca de algún sinónimo o sinónimos de esa expresión, mientras que otros son definitivamente considerados como no siendo, aun indirectamente, acerca de él o de ellos. Por otro lado, algunos enunciados son ordinariamente considerados como casos dudosos o limítrofes, lo que indica que en la clase de casos en cuestión no hay una línea divisoria clara y neta entre referencia indirecta y la ausencia de referencia indirecta. Los siguientes enunciados ilustran estos puntos.

a) El enunciado "La palabra 'bad' tiene un sinónimo en la lengua inglesa que está compuesto de cuatro letras" y "La palabra 'bad' tiene un sinónimo en la lengua inglesa", son ambas, indirectamente, acerca de la palabra 'evil': asumiendo que describen correctamente esa palabra.

b) En contraste con los dos enunciados precedentes, el enunciado "La palabra 'bad' está compuesta de tres letras" no es (ni siquiera) indirectamente acerca de la palabra 'evil'.

c) Finalmente, el enunciado "La palabra 'bad' tiene sinónimos" constituye, creo, un caso dudoso o limítrofe. Greo que algunas personas consideran que cae dentro de $a$ ), y otras considerarían que cae bajo $b$ ), supra, mientras que otras dudarían en subsumirlo bajo cualquiera de las dos.

Notemos en relación con esto que las anteriores y similares consideraciones no dan lugar a la suposición de que la referencia indirecta sea una cuestión de grado. El carácter escalar de un concepto dado, donde éste venga al caso, es un asunto completamente diferente, y no se sigue lógicamente de que el concepto sea o no de "textura abierta". Aún más, no encuentro ninguna evidencia, en el caso de las aplicaciones ordinarias de la palabra 'acerca 
de', de que la referencia indirecta sea susceptible de gradación. Ciertamente, hablamos de un enunciado o de un pasaje más largo de escritura, como un parágrafo, un capítulo de un libro o un libro, como siendo más acerca de una cosa que de otra. En forma similar hablamos de algunas obras de arte, tales como una ópera, un ballet, una novela o una pieza teatral dadas como siendo principal, primariamente, o en gran parte, acerca de un asunto particular. Pero en todos estos casos y en similares lo que tenemos en mente al hablar de esta manera es qué tanto dice el enunciado, pasaje u obra, acerca de una cosa particular, relativamente a lo que dice acerca de alguna otra cosa o cosas. En cada caso, ya sea directa o indirectmente, un enunciado, etc. es, simpliciter, acerca de algo o no es acerca de ello.

Con esto pasamos a enunciados de la forma " $U n$ ' $\mathrm{X}$ ' es un ' $\mathrm{YZ}$ " "que expresan el resultado de un analysis à la Moore. Tómese el enunciado, "La suegra es la madre del cónyuge", en el cual 'madre del cónyuge' expresa un analisans del concepto suegra. Este enunciado es directamente acerca de la entidad que es nombrada tanto por 'suegra' como por 'madre del cónyuge'; en forma más precisa, es directamente acerca de la identidad numérica de la entidad que nombra "suegra" y de la entidad que nombra "madre del cónyuge'. Pero no es también directamente acerca de las palabras 'suegra' y 'madre del cónyuge', ni tampoco es directa -o indirectamente- acerca del concepto suegra o madre del cónyuge, aunque sí implica lógicamente algo acerca de cada uno de ellos. ${ }^{13}$

"La suegra es la madre del cónyuge" no puede ser más que el enunciado de un análisis, una definición, porque no es directamente acerca de las palabras 'suegra' y 'madre del cónyuge' o cualesquiera otras palabras para ese caso. .Y no es trivial, aunque expresa un análisis correcto en parte porque afirma - de aquí que, para empezar, sea directamente acerca de- la identidad numérica del referente de dos diferentes, distintas expresiones verbales.

5) En el presente artículo hemos hablado constantemente acerca de los usos ordinarios de 'acerca de' en relación con oraciones y enunciados, u oraciones en uso. ${ }^{14}$ Nuestra pregunta final en este trabajo es acerca de si ordinariamente empleamos las palabras 'acerca de' exactamente de la misma manera en relación tanto con oraciones como con enunciados. La respuesta me parece ser negativa. Así, ordinariamente diríamos que el enunciado "Maine es pequeño", hecho en un contexto dado en una ocasión dada, normalmente es acerca de una de las cosas que son, o pueden ser, llamadas "Maine"; por ejemplo el estado de Nueva Inglaterra, mientras que la oración "Maine es pequeño" puede ser acerca de cualquier cosa llamada "Maine", ya sea el

13 Cf. mi propuesta solución a la Paradoja del Análisis en A Critical Study in Method, cap. 5 .

14 Sigo a P. F. Strawson al distinguir una oración y un enunciado que puede formarse a partir de ella en un contexto dado. 
estado en Nueva Inglaterra, un barco de guerra, o alguna otra cosa. Para establecer la diferencia en términos del concepto de referencia: un enunciado se refiere de hecho a una, o, a lo más, a unas cuantas cosas llamadas por un nombre particular (asumiendo, para empezar, que es un enunciado del tipo referencial); en tanto que una oración no se refiere de hecho a nada, pero puede ser usada para referirse a diferentes cosas en diferentes contextos, i. e., formando diferentes enunciados. Lo que hace que un enunciado particular como "Maine es pequeño" se refiera, digamos, al estado de Nueva Inglaterra y no a un barco de guerra norteamericano, etc., es la intención, del que habla o escribe, al hacer el enunciado en una ocasión particular. Se sigue que un individuo que hable o que escriba puede intentar referirse, simultáneamente, a dos o más cosas diferentes llamadas "Maine", con uno y el mismo enunciado. Este hecho es familiar como una forma de jugar con las palabras o de argucia. Será una instancia del primero si la intención o finalidad del que habla o escribe es producir un efecto humorístico, será, por otro lado, una instancia del segundo, si su intención es engañar al que escucha o lee.

Supongamos ahora que tomamos la oración "Paris es una ciudad grande" $(C)$, y el enunciado "Paris es una ciudad grande" $\left(C_{1}\right)^{*}$ proferido en un contexto dado. Una persona que no quiera ni jugar con las palabras ni engañar, al usar la palabra Paris en $C_{1}$ ], se referirá justamente a una cosa, digamos a la capital de Francia, o a una ciudad de Maine. Pero, ¿qué hay acerca de la oración $C$ ? ¿Acerca de qué puede ser? Por ejemplo, ¿puede ser, en algún sentido ordinario de "poder", acerca del amante de Helena de Troya, así como acerca de la capital de Francia y una ciudad de Maine? La respuesta es obviamente negativa, puesto que el amante de Helena no es una ciudad. Esto es, en el lenguaje ordinario, una oración sólo puede ser acerca de aquellas cosas que, si hacemos referencia a ellas, no convierten a la oración en autocontradictoria o absurda. Puesto que una oración tal como $C$ además de ser acerca de París, dice algo acerca de París, i. e., que es una ciudad grande, podemos plantear el asunto de la siguiente manera: una oración declarativa sólo puede referirse a aquellas cosas a las que sea aplicable, sin absurdo, lo que ella dice. '. . . es una ciudad grande' no puede ser acerca de Paris el amante de Helena, no puede ser un argumento de 'París...', no puede completarlo lógicamente sin absurdo. De aquí que en el sentido ordinario de esta palabra, $C$ no puede ser acerca del héroe troyano.

Una oración como $C$ debería ser claramente distiguida de una oración como "Una esfera es cilíndrica", la última es autocontradictoria tal y como está, como una oración, siempre que 'esfera' y 'cilíndrica' se usen en el sentido de "figura sólida engendrada por la revolución de un semicírculo alrededor de su diámetro" y "tener la forma de un sólido generado por una línea

* Recuérdese que en inglés no se distigue entre "Paris" y "Paris" [T.]. 
recta que se mueve paralelamente a sí misma y describe con sus extremos alguna curva fija, en especial un círculo", respectivamente, mientras que $C$ es una oración perfectamente consistente, a diferencia de "una esfera es cilíndrica", entendida en la forma anterior, sería autocontradictoria sólo en algunos enunciados formados de ella, esto es, sólo en contextos en los que se intentara referirse al amante de Helena o a cualquiera otra cosa que no fuera una ciudad. Pero nadie que sepa su sentido la afirmaria seriamente, i. e., intentaría de hecho referirse, a través de ella, digamos al amante de Helena. De aquí que, de hecho, no surja ningún problema en la práctica real.

Debe señalarse otra diferencia más entre las dos oraciones. "Una esfera es cilíndrica" será (directamente) acerca de esferas y la cualidad de ser cilíndrico aun en aquellos contextos en los que las palabras 'esfera' y 'cilíndrico' que contiene sean usadas en los anteriores sentidos. Mientras que, como hemos visto, $C$ no puede ser (directa o indirectamente) acerca de algo diferente a una ciudad en ningún contexto real o posible. Sin embargo, como con $C$, nadie afirmaría seriamente en ningún contexto real la oración "Una esfera es cilíndrica", en el sentido especificado; de aquí que, también en su caso, de hecho no surja ningún problema en la práctica real.

Haig Khatchadourian

University of Southern California

(Trad. de Raúl Quesada)

Los ANGeles 